\title{
Breast Cancer Early Detection Initiative: Don't Worry, Your Examination Means Safety, Holly Makkah, Saudi Arabia
}

\author{
Motair W, Alawi A, Alawi M, Khalaf M, Harraz M*, Qronfla M and Al-Solami SH \\ Department of Radiology, King Abd El-Aziz General Hospital (KAAH), Saudi Arabia \\ Submission: May 11, 2018; Published: May 31, 2018 \\ *Corresponding author: Harraz M, Department of Radiology, King Abd El-Aziz General Hospital (KAAH), Holly Makkah, Saudi Arabia, \\ Email: harrazharraz@live.com
}

\begin{abstract}
Breast cancer is the most common malignancy among women in Saudi Arabia. In Saudi Arabia, breast cancer cases are detected at relatively advanced stages (IIb \& III). Mammography ensures early diagnosis and a better chance for treatment and complete recovery. Programs for early diagnosis of breast cancer are still lacking in the Eastern Region countries. This paper reviews a governmental early detection initiative launched in September 2017 till March 2018 in Makkah of Saudi Arabia, in which 27 health centers, hospitals and public places were covered. Screening was offered to all women aged 40 years and above, a total of 15475 women were covered with this program. The number of cancers detected was 6, a cancer detection rate of 0.4 per 1000 women screened. The cancers detected had either no mass or the lesions were smaller than $2 \mathrm{~cm}$. The mean age of women with cancer was 51.4 (SD 7) years. A formal health education and screening program in Saudi Arabia is needed, for early detection to prevent breast cancer mortality, despite the controversies about efficacy of mammography screening.
\end{abstract}

Keywords: Mammography; Breast cancer; Screening

\section{Introduction}

Breast cancer is a global health problem, in Saudi Arabia, breast cancer is the commonest malignancy in women; it accounts for $25.8 \%$ of female malignancies as reported in the Saudi Cancer Registry [1]. There is an increase in the incidence of breast cancer in Saudi Arabia in the last few decades; with the median age at diagnosis of breast cancer is 49 years, but in the western societies (55-years-old) [2]. Most of the breast cancer cases in Saudi Arabia are detected at advanced stage than in developed countries, due to the lack of a national screening program, health education about cancer prevention and number of cultural barriers [2]. Screening programs are important in reducing breast cancer morbidity and mortality [3]. The mammography remains an effective means of early detection of breast cancer. In September 2017 the Saudi ministry of health, Makkah district, launched an initiative for early detection of breast cancer in the Makka Province of Saudi Arabia which is continuing to March 2018. This paper reviews the scheme and presents the results from this program, focusing on uptake and cancer detection rates.

\section{Methods}

This study includes data from September 2017 to March 2018. Approval for this study was obtained from the institutional review board of King Abd El-Aziz General Hospital (KAAH), Makkah, Saudi Arabia. Prior to and during the screening program multiple public outreach activities were undertaken by female volunteers, who included doctors, nurses and students. Lecturers and interactive sessions about breast cancer awareness and screening were carried out in universities, schools, residential compounds and shopping malls. Information brochures in Arabic and English were also distributed. Similar activities were undertaken by male volunteers. These activities were not a one-time effort but continued throughout the initiative. Also 7 general hospitals were covered by the screening program (King Abd El-Aziz, Ibn sinaa, maternity and children, Herra, King Faisal, Al-kamel \& Khelis hospitals).

All women were self-referred with no formal invitation was given for screening. No upper limit was set for screening. Women with a strong family history of breast cancer were offered earlier screening, at age 35 years. The exclusion criteria were age less than 35 years, pregnancy, lactation, symptomatic patient, and suspicious findings on clinical examination. Data from all women who enrolled in the screening program were included in this study. Verbal consent for participation was taken from all of them. 


\section{Data collection and screening}

Prior to mammogram examination, a detailed history was taken from each woman. The history included demographic data, age of menarche, age at first child and history of breastfeeding. A trained nurse examined the woman and recorded her weight and height. Figure 1 summarizes the work-up plan followed during the screening program. A standard full-field digital mammography was done for all women. Standard craniocaudal and mediolateral oblique views of each breast were obtained. Symptomatic women and those with positive findings were referred to breast clinics for diagnostic workup. All mammograms were read by a senior radiologist with more than 25 years' experience of breast imaging. A random second reading was done by radiologists with breast imaging experience ranging from 10 to 15 years. The standard American College of Radiology (ACR) lexicon and Breast Imaging Reporting and Data System (BI-RADS) categories were used in reporting. In case of a difference in BI-RADS grading, the higher BIRADS grade was recorded. All mammograms were initially reported as BI-RADS 0 (incomplete), BI-RADS 1 or BI-RADS 2.

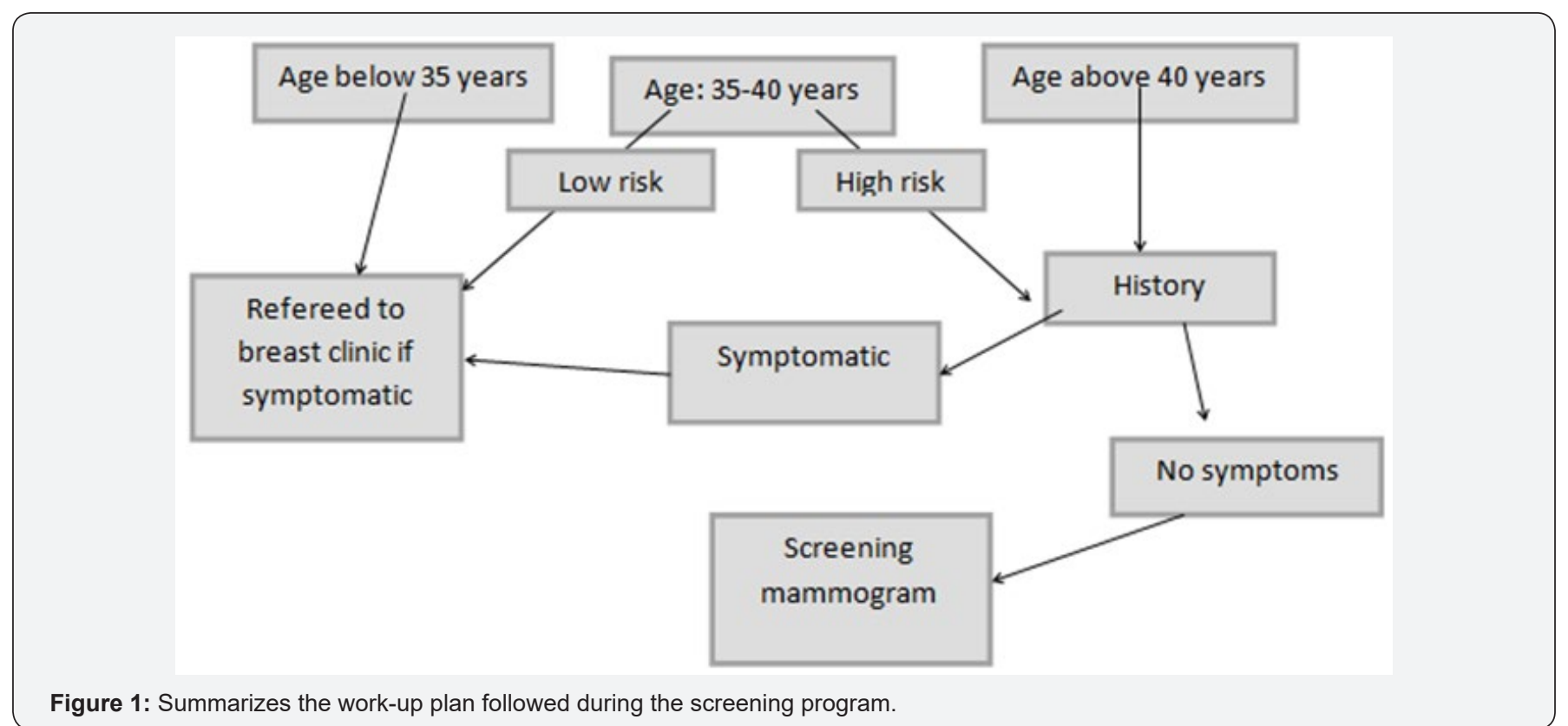

\section{Post-screening and follow-up}

Women with BI-RADS category 1 and 2 were reassured and were instructed to do routine annual screening. Those with BI-RADS category 0 were evaluated by additional views and/or ultrasound as well as MRI if indicated. Following further evaluation of the BI-RADS 0 cases they were further characterized into BI-RADS 1 to 5 . Women with BI-RADS 3 were given short-term follow-up by ultrasound or mammogram as required. Those with BI-RADS 4 and 5 were biopsied either under ultrasound or stereotactic guidance, or also given a surgical consultation. The follow-up examinations were done mainly in King Abd El-Aziz general Hospital, Makkah, further imaging including additional mammographic projections, ultrasound evaluation or magnetic resonance imaging were done.

\section{Results}

From 15475 women (which is the target number in this phase of the initiative), 1217 women attended and underwent screening (uptake rate of $8 \%$ ). The specified age for inclusion was above 40 years old. Most $(540 / 1217,44 \%)$ of the screened ladies were premenopausal, 255/1217 (22\%) were peri-menopausal and 422/1217 (34\%) were postmenopausal. Out of 1217 mammograms, 169 required further assessment with breast ultrasound since 145 were BIRAD III, 21 were BIRAD IV and 3 were BIRAD V (Recall Rate 14\%). After ultrasound, 6 cases required biopsy (Biopsy Rate 2.7\%). In 25 cases, pathology results were of different benign breast conditions like fibro-adenomas, fibrocystic changes and benign epithelial hyperplasia. 6 cases were confirmed pathologically to be malignant; 4 cases of invasive ductal carcinoma, grade II (IDC, II), 1 cases of ductal carcinoma insitu (DCIS) and 1 case of invasive lobular carcinoma (ILC). The average age of cancer cases was 51.4 years ( $\mathrm{SD} \pm 7$ ); 2 of them were peri-menopausal and 4 were postmenopausal. None of the cancer cases had past history of breast disease or breast biopsy. Family history of breast cancer was positive in 3 cases. They were referred to the tertiary centers to receive the required surgical/oncology treatment.

\section{Discussion}

The Saudi Arabia has experienced a rise in the number of breast cancer cases. As such, there is an urgent need for prevention and early diagnosis of this disease. Formal national screening programs are lacking in the Arab region, despite its efficacy in the reduction of mortality from breast cancer. The World Health Organization has stressed in its report for the Eastern Region for the importance of early detection to prevent breast cancer mortality. Mammography remains the mainstay for the early diagnosis of breast cancer [1]. 
The previous studies that documented breast cancer screening participation rates found them to be low for women throughout the Arab region; knowledge of the benefits of breast cancer screening is an important determinant of breast cancer screening behavior [4]. The median age reported at diagnosis of breast cancer in Saudi Arabia is 49 years compared to 51.4 years in our study. The demographic characteristics are different from the other characteristics reported in Western women, so western mammographic screening guidelines are inappropriate for Saudi women. In every country, mammography screening guidelines should be done according to the local parameters and these include breast cancer incidence, age groups affected, risk factors and healthcare resources [5]. It was reported that initiating screening at age 40 with either annual or biennial intervals is associated with a $3 \%$ median reduction in breast cancer mortality. A screening of a younger population with a higher breast density is associated with increased recall and biopsy rates [6]. Saudi women are encouraged to be self-aware in order to be able to seek medical opinion appropriately but we don't have local data on the effect of breast self-examination (BSE) on early cancer detection. Alsaif et al. [7] found a significant relation between higher levels of education and BSE practice at a study on Saudi nursing students and BSE. Aboulfotouh et al. [8] found that only $41.6 \%$ reported ever practicing BSE and $21 \%$ only performed it regularly, in his study about the practice of BSE among adult Saudi female employees, working at King bdulaziz Medical City, Riyadh, Saudi Arabia and their non-working adult female family members. Screening is a continuous process that should be associated with minimum obstacles to encourage ladies to come willingly for the next cycle. Our concern and aim is to have and maintain a smooth screening journey that provides Saudi ladies with the required optimum health service.

\section{Conclusion}

Saudi women accepted mammographic screening willingly. Breast-cancer screening has to be integrated into an optimallyfunctioning, properly staffed and well-equipped health care system.

\section{Ethical Approval}

Approval of the medical ethics committee was obtained for publication.

\section{References}

1. Mokdad AH, Jaber S, Aziz MIA, Al Buhairan F, AlGhaithi A, et al. (2014) The state of health in the Arab world, 1990-2010: an analysis of the burden of diseases, injuries, and risk factors. Lancet 383(9914): 309320.

2. Ferlay J, Soerjomataram I, Ervik M, Dikshit R, Eser S, et al. (2012) Cancer incidence and mortality worldwide. International Agency for Research on Cancer, Lyon, France.

3. Ibrahim EM, Zeeneldin AA, Sadiq BB, Ezzat AA (2008) The present and the future of breast cancer burden in the Kingdom of Saudi Arabia. Med Oncol 25(4): 387-393

4. Amin TT, Al Mulhim ARS, Al Meqihwi A (2009) Breast cancer knowledge, risk factors and screening among adult Saudi women in a primary health care setting. Asian Pac J Cancer Prev 10(1): 133-138.

5. Bray F, Ren JS, Masuyer E, Ferlay J (2013) Global estimates of cancer prevalence for 27 sites in the adult population in 2008. Int J Cancer 132(5): 1133-1145.

6. Cappellani A, Vita MD, Zanghì A, Cavallaro A, Piccolo G, et al. (2013) Prognostic factors in elderly patients with breast cancer. BMC Surg 13(Suppl 2): S2

7. Alsaif AA (2004) Breast self-examination among Saudi female nursing students in Saudi Arabia. Saudi Med J 25(11): 1574-1578.

8. Abolfotouh MA, BaniMustafa AA, Mahfouz AA, Al Assiri MH, Al Juhani $A F$, et al. (2015) Using the health belief model to predict breast selfexamination among Saudi women. BMC Public Health 15: 1163.

\section{Your next submission with Juniper Publishers will reach you the below assets}

